University of Wollongong

Research Online

Faculty of Engineering and Information

Faculty of Engineering and Information

Sciences - Papers: Part A

Sciences

January 2016

\title{
Advanced point of common coupling voltage controllers for grid-connected solar photovoltaic (PV) systems
}

\author{
Brian Perera \\ University of Wollongong, bkp389@uowmail.edu.au \\ Philip Ciufo \\ University of Wollongong, ciufo@uow.edu.au \\ Sarath Perera \\ University of Wollongong, sarath@uow.edu.au
}

Follow this and additional works at: https://ro.uow.edu.au/eispapers

Research Online is the open access institutional repository for the University of Wollongong. For further information contact the UOW Library: research-pubs@uow.edu.au 


\title{
Advanced point of common coupling voltage controllers for grid-connected solar photovoltaic (PV) systems
}

\author{
Abstract \\ The voltage rise of the low voltage (LV) power distribution grid to which multiple solar photovoltaic (PV) \\ systems are integrated is a critical technical problem that should be addressed. With PV systems that are \\ integrated to the LV power distribution grid (with an $\$ R$ S to $\$ X \$$ ratio greater than unity) via voltage source \\ converters, the opportunity exists to regulate the respective point of common coupling (PCC) voltages by \\ dynamically controlling the active and reactive power response of PV systems. In this paper, two closed- \\ loop controllers that are able to regulate the PCC voltage by dynamically controlling the active and \\ reactive power response of the PV system are presented. The design methodology is presented with \\ considerable detail. The plant model of each controller is derived and the design procedure of each \\ controller is explained in detail. By combining the dynamic active and reactive power controllers proposed \\ in this paper, two novel operating strategies for PV systems, fixed minimum power factor operation and \\ fixed maximum apparent power operation, are introduced. The latter operating strategy has been \\ identified as the most efficient way of regulating the PCC voltage of a PV system. The simulation results \\ and experimental validation confirm the accuracy of the derived plant models, the robustness of the \\ designed controllers and the feasibility of implementing the proposed novel operating strategies in PV \\ systems.
}

\section{Keywords}

systems, advanced, point, pv, photovoltaic, solar, common, connected, coupling, grid, controllers, voltage

\section{Publication Details}

B. Perera, P. Ciufo \& S. Perera, "Advanced point of common coupling voltage controllers for gridconnected solar photovoltaic (PV) systems," Renewable Energy, vol. 86, pp. 1037-1044, 2016. 


\title{
Advanced Point of Common Coupling Voltage Controllers for Grid-Connected Solar Photovoltaic (PV) Systems
}

\author{
Brian Perera, Philip Ciufo*, Sarath Perera* \\ Australian Power Quality and Reliability Centre, School of Electrical, Computer, and \\ Telecommunications Engineering, University of Wollongong, Wollongong, New South Wales \\ 2522, Australia
}

\begin{abstract}
The voltage rise of the low voltage (LV) power distribution grid to which multiple solar photovoltaic (PV) systems are integrated is a critical technical problem that should be addressed. With PV systems that are integrated to the LV power distribution grid (with an $R$ to $X$ ratio greater than unity) via voltage source converters, the opportunity exists to regulate the respective point of common coupling (PCC) voltages by dynamically controlling the active and reactive power response of $\mathrm{PV}$ systems. In this paper, two closed-loop controllers that are able to regulate the PCC voltage by dynamically controlling the active and reactive power response of the PV system are presented. The design methodology is presented with considerable detail. The plant model of each controller is derived and the design procedure of each controller is explained in detail. By combining the dynamic active and reactive power controllers proposed in this paper, two novel operating strategies for PV systems, fixed minimum power factor operation and fixed maximum apparent power operation, are introduced. The latter operating strategy has been identified as the most efficient way of regulating the PCC voltage of a PV system. The simulation results and experimental validation confirm the accuracy of the derived plant models, the robustness of the designed controllers and the feasibility of implementing the proposed novel operating strategies in PV systems.
\end{abstract}

Keywords: voltage control; photovoltaic system; point of common coupling; voltage source converter; voltage regulation

\footnotetext{
*Corresponding author. Tel +61 2 42213406, Fax. +61 242213236

Email addresses: bkp389@uowmail.edu.au (Brian Perera), philip_ciufo@uow.edu.au (Philip Ciufo), sarath@uow.edu.au (Sarath Perera)

${ }^{1}$ NOTICE: this is the authors' version of a work that was accepted for publication in Renewable Energy. Changes resulting from the publishing process, such as peer review, editing, corrections, structural formatting, and other quality control mechanisms may not be reflected in this document. Changes may have been made to this work since it was submitted for publication. A definitive version was subsequently published in Renewable Energy, [vol. 86, pages 1037-1044 (Feb. 2016)] http://dx.doi.org/10.1016/j.renene.2015.09.028
} 


\section{Introduction}

The integration of multiple solar photovoltaic (PV) systems to the low voltage power distribution grid (herein after referred to as the grid) has led to a grid voltage rise problem [1-3]. The grid voltage may exceed the specified highest voltage of $230 \mathrm{~V}+10 \%$ (herein after referred to as $U_{\max }$ ) as in the Australian Standard AS4777.2 [4], especially in situations when the power generation from PV systems is at a peak and the load on the grid is at a minimum. In such a situation, as per [4], where the point of common coupling (PCC) voltage is above $U_{\max }$, the PV systems should be automatically disconnected. The disconnection of PV systems leads to loss of energy yield from the installed PV systems and income for the resource owner.

$\mathrm{PV}$ systems are integrated to the grid via power electronic converters and, more specifically, often via voltage source converters (VSC) [5], [6]. A VSC is capable of operating in all four quadrants when designed appropriately. In the grid, the sensitivity of a node voltage to active and reactive power is a function of the grid impedance seen at that particular node. Therefore, the opportunity exists for PV systems integrated to the grid via VSCs to regulate the respective PCC voltage by dynamically controlling the active and reactive power injected and minimise the grid voltage rise.

The static reactive power control methodologies such as constant and variable power factor have been identified as inefficient as these methods may cause additional reactive power flow in the grid even when the grid voltage is well within the limits and may lead to overloading of the distribution transformer [1]. A droop based reactive power control method $(U(Q))$ has been proposed in [7] which minimises the drawbacks of the static reactive power control methodologies. Strategies devised by [8], [9] and [10] exploit such a droop-based control scheme, but require systemwide communications in order to ensure a coordinated approach to reactive power control and active power curtailment. In order to implement the $U(Q)$ reactive power control method effectively, grid specific studies or additional decision making control algorithms such as fuzzy logic based adaptive controller presented in [11] and [12] are necessary. The PCC voltage of a PV system can be regulated by a closed-loop controller that dynamically controls the reactive power response of the PV system. Such a controller has been identified as an effective way of regulating PCC voltage with reactive power [1].

The ability to regulate the PCC voltage with a closed-loop controller that dynamically regulates the reactive power injected to the grid by the PV system is limited. The limitation is mainly due to the limited rating of the VSC that interfaces the PV system to the grid. When the PV system is injecting a certain amount of active power to the grid, only the excess capacity of the VSC, if any, can be used to inject/absorb reactive power. The available limited reactive power capacity of the PV system at a given time may not be adequate to regulate the PCC voltage at $U_{\max }$ or at any other specified voltage reference depending on the voltage sensitivity of the PCC. In such a situation active power curtailing is an option to control the PCC voltage in the absence of energy storage systems $[3,13,14]$. Similar to closedloop reactive power control of a PV system, closed-loop active power control can be proposed as an efficient way of regulating PCC voltage of a PV system with active power. The authors of [15] have developed a system of active power curtailment 
for use in networks which have very high $R / X$ ratios. This scheme results in an unequal curtailment between the devices connected to a highly resistive $(R / X>7)$ distribution network and consequently an unequal loss of income. A variation to the control is introduced in order to rectify this situation, but the result is a reduction in the overall active power that is injected to the grid.

In the published literature, little attention has been given in providing adequate technical details and guidelines for practically implementing closed-loop controllers that control the active and reactive power response of a PV system in order to regulate the PCC voltage of the PV system. In some cases, [12], the reported design is subject to patent and therefore difficult for other researchers to access. In other instances, the technical details required to replicate the controller are not presented. In this paper, a detailed design procedure of closed-loop active and reactive power controllers that are capable of dynamically regulating the PCC voltage of a gridconnected PV system is provided.

Large variations in grid impedance can affect the performance of PCC voltage controllers. Therefore, the sensitivity of the developed controllers to grid impedance variations are discussed together with their respective controller gain values. The design strategies required in order to account for the wide range of grid impedances is not considered in the current research. Instead, both the simulation and experimental analyses use typical grid impedances as defined in [16].

The contributions of the current research are the development of an active power controller that can be used to control the PCC voltage of a PV system dynamically and two novel operating strategies developed by combining the operation of closedloop active and reactive power controllers. In this research, simulation results of a PV system that is integrated with closed-loop active and reactive power controllers are presented. Experimental results are provided to demonstrate the robustness of the designed closed-loop active and reactive power controllers and the feasibility of implementing novel operating strategies for grid-connected PV systems proposed in the current research.

The technical scope of this paper is limited to illustrate the design of dynamic active and reactive power controllers in detail and the introduction of the aforementioned operating strategies for a grid-connected PV system. The effects of dynamic behaviour of loads and other inverter interfaced energy sources connected to the grid are not considered in this paper. Evaluation of the performance and interaction of the dynamic active and reactive power controllers and the proposed novel operating strategies under external disturbances are future work arising from this research. Sufficient design detail has been presented that will enable other researchers to extend and develop the concepts outlined in this paper.

The remainder of this paper is organised as follows: the simplified model of the grid that is considered in designing the closed-loop active and reactive power controllers are described in Section 2, and in Section 3, the design procedure of the closed-loop reactive power controller is presented. The detailed design procedure of the closed-loop active power controller is illustrated in Section 4. In Section 5, two novel operating strategies are proposed by combining both active and reactive power controllers. Finally, in Section 6, experimental results of the proposed controllers and operating strategies are presented followed by conclusions in Section 7 . 


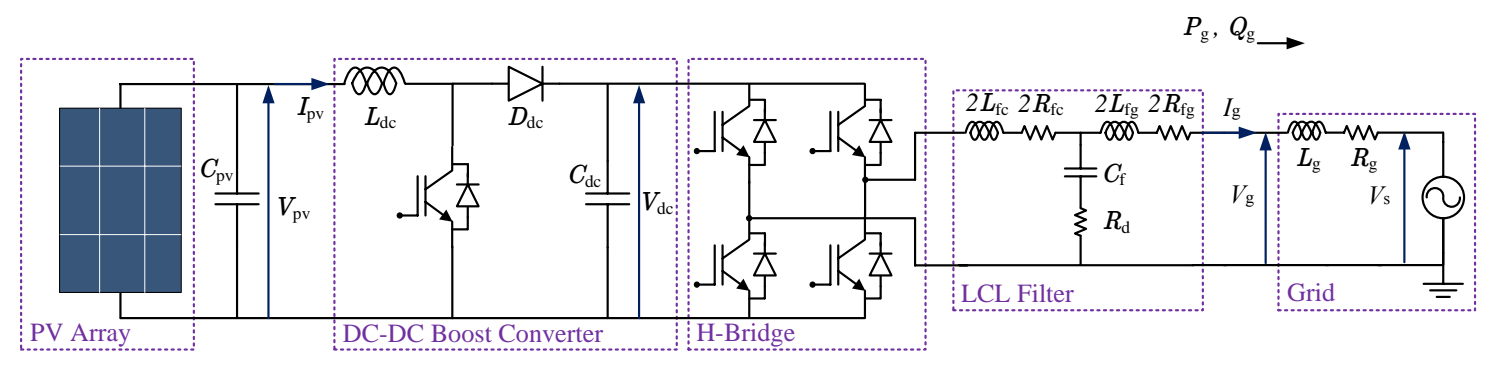

Figure 1: Grid-connected PV system with a two-stage converter

\section{Simplified model of a distribution feeder}

The modelled PV system shown in Fig. 1 consists of component models of a PV array, a DC-DC boost converter, a voltage source converter (VSC), an LCL filter, and a model of a power distribution grid.

The active and reactive power controllers are designed without considering the effects of the dynamic behaviour of loads and other inverter interfaced energy sources connected to the grid. Hence a simplified model of a power distribution feeder is considered in the design as shown in Fig. 2. The grid is modelled with an equivalent Thèvenin voltage source (of which the rms voltage at the terminal is $V_{\mathrm{s}}$ ) that is connected in series with a resistor, $R_{\mathrm{g}}$, and a reactance, $X_{\mathrm{g}}$. The rms voltage at the PCC of the PV system is $V_{\mathrm{g}}$. The reactance $X_{\mathrm{f}}$ represents the LCL filter of the PV system. The PV system is considered to inject current $I_{\mathrm{g}}$ to the grid resulting in real power of $P_{\mathrm{g}}$ and reactive power of $Q_{\mathrm{g}}$ delivered to the grid.

The reference impedance for low voltage public supply systems given in [16] for electrical apparatus testing purposes is used to model the grid impedance. Hence the grid impedance of the simplified model of the power distribution feeder is $R_{\mathrm{g}}+j X_{\mathrm{g}}=$ $(0.4+j 0.25) \Omega$. The $R / X$ ratio of the considered grid is approximately 1.6. Hence an opportunity exists to regulate the $\mathrm{PCC}$ voltage by dynamically regulating the active and reactive power response of the PV system.

\section{PCC voltage regulation with the dynamic reactive power controller - (PCC $V_{\mathrm{Q}}$ controller)}

The closed-loop PCC voltage controller that dynamically controls the reactive power response of the PV system (herein after referred to as the PCC $V_{Q}$ controller) described in detail in [17] is summarised in this Section.

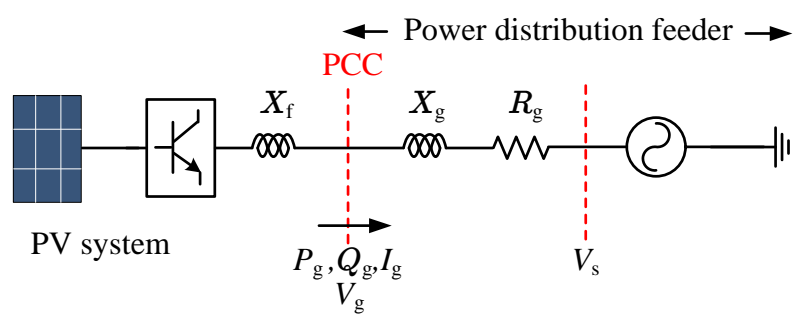

Figure 2: Simplified model of a power distribution feeder. 


\subsection{Control plant model of the PCC $V_{Q}$ controller}

The control plant model of the PCC $V_{Q}$ controller, $G_{V_{\mathrm{gQ}}}(s)$ can be derived as given in (1) [17]. The plant model is derived by applying a time discriminated decoupling mechanism as suggested in [18] to decouple the PCC $V_{Q}$ controller from the rest of the control system of the PV system. In (1), $L_{\mathrm{g}}=X_{\mathrm{g}} / \omega$ where $\omega$ is the nominal power frequency. $\Delta V_{\mathrm{gm}}$ is the change in the peak value of the PCC voltage $V_{\mathrm{g}}$, due to $\Delta I_{\mathrm{gq}}$ change in the peak value of the reactive current absorbed by the PV system.

$$
G_{V_{\mathrm{gQ}}}(s)=\frac{\Delta V_{\mathrm{gm}}(s)}{\Delta I_{\mathrm{gq}}(s)}=-\omega L_{\mathrm{g}}
$$

\section{2. $P C C V_{Q}$ controller}

The closed-loop system of the PCC $V_{Q}$ controller is shown in Fig. 3(a) where $V_{\mathrm{gm}}$ is the peak value of the PCC voltage $V_{\mathrm{g}}$, and $V_{\mathrm{gm}}$ can be obtained through the phase-locked-loop (PLL) of the PV system [19]. $V_{\mathrm{gm} 0}$ is the peak value of the reference voltage of the PCC. $Q_{\text {ref }}$ is the reactive power reference. $I_{\text {qref }}$ is the peak value of the reactive current reference of the closed-loop current controller, $G_{\mathrm{cc}}(s)$ is described in detail in [19]. $I_{\mathrm{gq}}$ is the peak value of the reactive current output of the PV system and $G_{V_{\mathrm{gQ}}}(s)$ is the plant model of the PCC $V_{Q}$ controller.

An integrator with a gain is a suitable compensator for the PCC $V_{Q}$ controller. Further, the gain of the integrator, $k_{\mathrm{pq}}$, as shown in Fig. 3(a), can be determined to achieve the steady-state of the PCC $V_{Q}$ controller within a suitable time delay using the reference grid impedance as mentioned in Section 2, assuming $G_{\mathrm{cc}}(s)=1$ [17]. In this case, $1 \mathrm{~s}$ was chosen as the settling time.

Simulation results obtained by integrating the PCC $V_{Q}$ controller with the simulation model of the PV system in [19] are shown in Fig. 3(b). In this simulation study, the PCC voltage was controlled only by the PCC $V_{Q}$ controller by absorbing reactive power from the grid. Active power injection of the PV system is not impacted by the operation of PCC $V_{Q}$ controller since active and reactive power controllers are decoupled. The graphs in the figure illustrate the dynamic performance of the PCC $V_{Q}$ controller under an external disturbance; a step change in the network voltage, represented by $V_{s}$ in Fig. 2. In the simulation model, $V_{\text {gm0 }}$ was set as $\sqrt{2} U_{\max }$ and the PCC $V_{Q}$ controller was configured to activate only when $V_{\mathrm{gm}}>V_{\mathrm{gm} 0}$. At time $t=1 \mathrm{~s}$, a step change in the network voltage was applied. The applied network voltage change has caused the PCC voltage to rise above the reference voltage. Hence, the PCC $V_{Q}$ controller was activated and the controller was able to regulate the PCC voltage at the reference level set within $1 \mathrm{~s}$.

\section{PCC voltage regulation with the dynamic active power controller - (PCC $V_{P}$ controller)}

The detailed design procedure of the closed-loop PCC voltage controller that dynamically controls the active power response of a grid-connected PV system (herein after referred to as the PCC $V_{P}$ controller) is explained in this Section. 


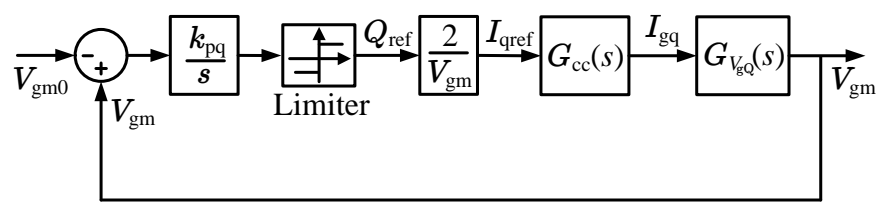

(a) Control block diagram of the PCC $V_{Q}$ controller.

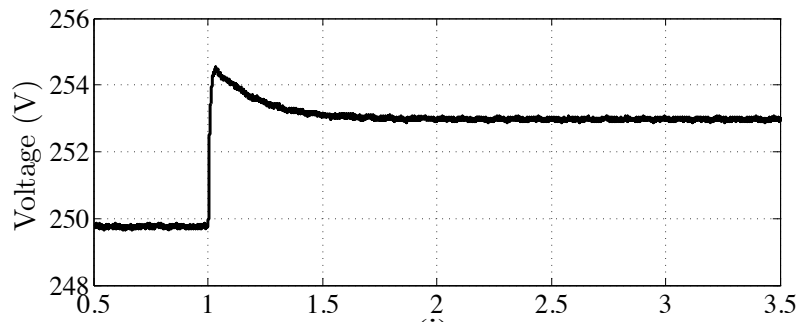

(i)

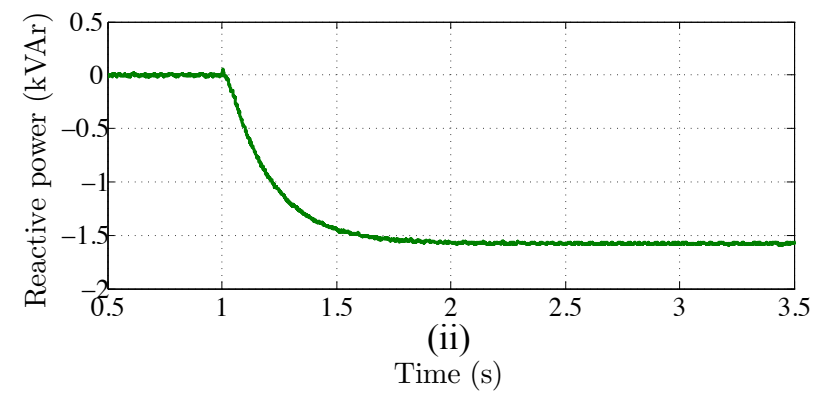

(b) Dynamic performance of the PCC $V_{Q}$ controller following a disturbance; (i) rms voltage of the PCC $\left(V_{\mathrm{g}}\right)$ and (ii) reactive power absorbed from the grid $\left(Q_{\mathrm{g}}\right)$ by the PV system.

Figure 3: Block diagram and performance of the PCC $V_{Q}$ controller

\subsection{Decoupling of the PCC $V_{P}$ controller}

In the grid-connected PV system shown in Fig. 2, the amount of active power injected is determined by the response of the controller of the DC-DC boost converter. The reference to the controller of the DC-DC boost converter is provided by a maximum power point tracking (MPPT) algorithm. In the PV system, the active power flow is controlled by the DC-link voltage controller and the current controller. The accurate command tracking of the control system of the PV system and also decoupling of control systems (as suggested in [18]) where applicable are realised by properly selecting the response time of the controllers [17], [19]. Similarly, the response time of the PCC $V_{P}$ controller is discriminated in order to decouple the controller from the rest of the control system that is associated in active power transfer of the PV system.

\subsection{Control plant model of the PCC $V_{P}$ controller}

The control plant model of the PCC $V_{P}$ controller is derived based on the simplified model of the power distribution feeder shown in Fig. 2. Further, since the PCC $V_{P}$ controller is designed to have a long response time compared to that of the current controller of the PV system, steady-state operation of the network shown in Fig. 2 is considered while deriving the control plant model. 


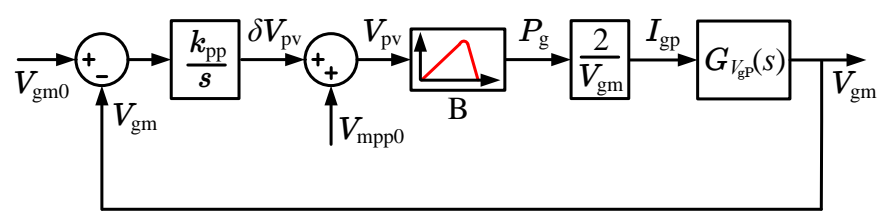

Figure 4: Control block diagram of the PCC $V_{P}$ controller.

If KVL is applied to the circuit in Fig. 2, (2) can be derived.

$$
\vec{V}_{\mathrm{g}}-\vec{V}_{\mathrm{s}}=\left(R_{\mathrm{g}}+j X_{\mathrm{g}}\right) \vec{I}_{\mathrm{g}}
$$

A small change in the peak value of the active current injected to the grid by the PV system, $\Delta I_{\mathrm{gp}}$ is considered. The change in the peak value of $V_{\mathrm{g}}$ because of the voltage drop $j X_{\mathrm{g}} \Delta I_{\mathrm{gp}}$ can be assumed negligible since $V_{\mathrm{gm}}>\left|X_{\mathrm{g}} \Delta I_{\mathrm{gp}}\right|$ ( $V_{\mathrm{gm}}$ is the peak value of $V_{\mathrm{g}}$ ). Hence, the phase angle deviation between $V_{\mathrm{g}}$ and $V_{\mathrm{s}}$ because of the small change in the active current injected to the grid can be disregarded. Therefore, for a small change in active current injected to the grid by the PV system, (3) can be derived from (2). In (3), $\Delta V_{\mathrm{gm}}$ is the change in the peak value of $V_{\mathrm{g}}$ because of $\Delta I_{\mathrm{gp}}$.

$$
\Delta V_{\mathrm{gm}} \approx R_{\mathrm{g}} \Delta I_{\mathrm{gp}}
$$

The control plant model of the PCC $V_{P}$ controller can be obtained as given in (4) by applying the Laplace transformation to (3).

$$
G_{V_{\mathrm{gP}}}(s)=\frac{\Delta V_{\mathrm{gm}}(s)}{\Delta I_{\mathrm{gp}}(s)} \approx R_{\mathrm{g}}
$$

\section{3. $P C C V_{P}$ controller}

The proposed closed-loop PCC $V_{P}$ controller is shown in Fig. 4. This controller regulates the $\mathrm{PCC}$ voltage by controlling the active power injected by the PV system in situations where $V_{\mathrm{gm}}>V_{\mathrm{gm} 0}$. In such a situation, the operating point of the PV system deviates from the maximum power point (MPP) and the PV system is operated at a lower power level. Therefore, when the PCC $V_{P}$ controller is enabled, MPP tracking is not needed. Hence the MPPT tracking algorithm is configured to deactivate upon the activation of the controller. In Fig. $4, V_{\mathrm{mpp} 0}$ is the voltage at the maximum power point (MPP) where the PV array was operating before the PCC $V_{P}$ controller commences regulating the PCC voltage. $G_{V_{\mathrm{gP}}}(s)$ is the control plant model of PCC $V_{P}$ controller.

In Fig. 4, Block B represents the mathematical relationship between the active power injected to the grid by the PV system, $P_{\mathrm{g}}$ and the operating voltage of the $\mathrm{PV}$ array, $V_{\mathrm{pv}}$. The mathematical relationship can be derived by considering the characteristic curves of the PV array. A typical terminal voltage and power characteristic curve of a PV array is shown in Fig. 5 for a selected weather condition when the solar irradiance level is $1200 \mathrm{~W} / \mathrm{m}^{2}$ and the ambient temperature is $30{ }^{\circ} \mathrm{C}$. The voltage at the MPP, $V_{\mathrm{mpp} 0}=290 \mathrm{~V}$ and the power available at the MPP, $P_{\mathrm{mpp}}$ is approximately $4.9 \mathrm{~kW}$. The open circuit voltage of the PV array, $V_{\mathrm{oc}}$ is about $360 \mathrm{~V}$. $V_{\mathrm{pv}}$ is an arbitrary voltage across the PV array at a given time and is controlled by the DC-DC boost converter controller. $P_{\mathrm{g}}$ is the amount of active power injected to 


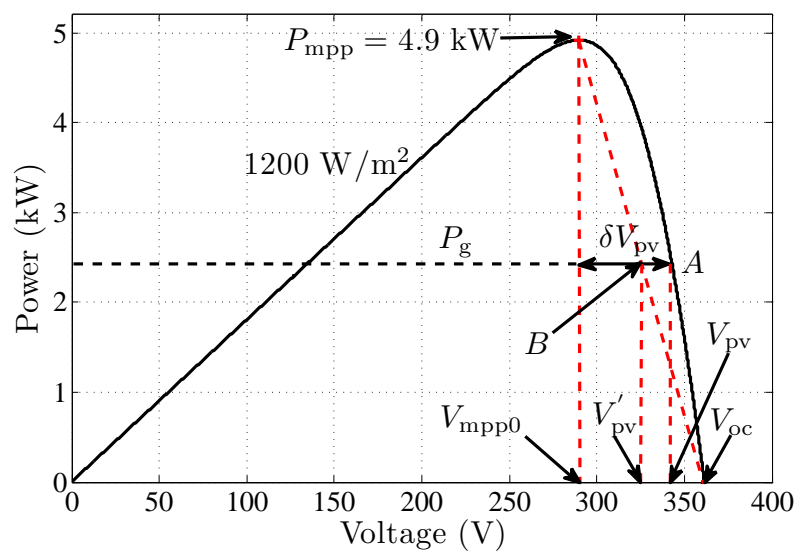

Figure 5: $V P$ characteristics of the $\mathrm{PV}$ array.

the grid by the PV system if the voltage across the PV array is controlled at $V_{\mathrm{pv}}$, assuming system losses are negligible. Such control action leads the operating point of the PV array to be at $A$. In Fig. $4, I_{\mathrm{gp}}$ is the peak value of the active current injected to the grid. The closed-loop current controller of the PV system is not shown in Fig. 4 as it is considered to be a pure gain equal to unity.

The PCC $V_{P}$ controller is designed in such a way that the operating point of the PV array is maintained at an operating point to the right-hand side of the line $P_{\mathrm{mpp}} V_{\mathrm{mpp} 0}$ in Fig. 5. In the PCC voltage controller shown in Fig. $4, \delta V_{\mathrm{pv}}(>0)$ is the voltage added to $V_{\mathrm{mpp} 0}$ in order to curtail the active power injected by the PV system to control the PCC voltage by moving the operating point of the PV array to a lower power level, $P_{\mathrm{g}}\left(<P_{\mathrm{mpp}}\right)$. The voltage $\delta V_{\mathrm{pv}}$ that should be added to $V_{\mathrm{mpp} 0}$ to control the PCC voltage is the output of the compensator. Since $\delta V_{\mathrm{pv}}$ is added to $V_{\mathrm{mpp} 0}$ and also $\delta V_{\mathrm{pv}}>0$, when active power is curtailed, $V_{\mathrm{pv}}>V_{\mathrm{mpp} 0}$. Thereby a stable operating point is always maintained in the PV array when the active power is dynamically controlled to regulate the PCC voltage.

In the characteristic curve of the PV array shown in Fig. 5, only the section $V_{\mathrm{mpp} 0} P_{\mathrm{mpp}} A V_{\text {oc }}$ is of the interest since the PCC $V_{P}$ controller ensures that the operating point of the PV array to be within $P_{\mathrm{mpp}} A V_{\mathrm{oc}}$. The curve section $P_{\mathrm{mpp}} A V_{\mathrm{oc}}$ of the characteristic curve of the PV array is non-linear. In order to derive an approximated linear expression for the Block $\mathrm{B}$, the curve section $P_{\mathrm{mpp}} A V_{\mathrm{oc}}$ of the characteristic curve of the $\mathrm{PV}$ array is approximated to the straight line $P_{\mathrm{mpp}} V_{\mathrm{oc}}$. With the stated approximation, the operating point of the PV array becomes $B$ when injecting an amount of active power equals to $P_{\mathrm{g}}$ to the grid and the voltage of the PV array at $B$ is $V_{\mathrm{pv}}^{\prime}$. A linear expression for the Block $\mathrm{B}$ can be derived using triangles $V_{\mathrm{mpp} 0} P_{\mathrm{mpp}} V_{\mathrm{oc}}$ and $V_{\mathrm{pv}}^{\prime} B V_{\mathrm{oc}}$ in Fig. 5, such that:

$$
P_{\mathrm{g}}=\underbrace{\frac{-P_{\mathrm{mpp}}}{V_{\mathrm{oc}}-V_{\mathrm{mpp} 0}}}_{m} V_{\mathrm{pv}}^{\prime}+\underbrace{\frac{P_{\mathrm{mpp}} V_{\mathrm{oc}}}{V_{\mathrm{oc}}-V_{\mathrm{mpp} 0}}}_{C},
$$

and

$$
P_{\mathrm{g}}=m V_{\mathrm{pv}}^{\prime}+C
$$

The voltage and power at the MPP, and the open circuit voltage of a PV panel at 


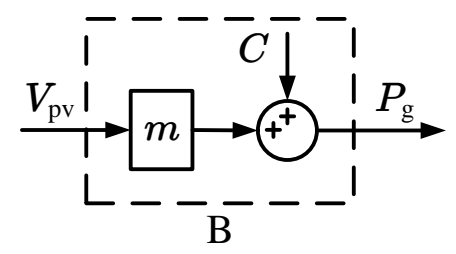

Figure 6: A detailed representation of Block B in Fig. 4.

rated conditions is generally available from the data sheet of the PV panel. Hence, in general, the data sheet of the PV panel can be used to determine the parameter $m$ in Block B. For the given operating conditions of the PV array described by Fig. $5, m=-70 \mathrm{~W} / \mathrm{V}$ and $C=25200 \mathrm{~W}$ as given in (6). Block B in Fig. 4 can be represented as illustrated in Fig. 6 with the use of (6). The effect of the linear approximation that is made while deriving (6) is disregarded when representing the Block B in detail in Fig. 6, so that $V_{\mathrm{pv}}^{\prime}$ in (6) equals to $V_{\mathrm{pv}}$. When designing the PCC $V_{P}$ controller, the loop gain, $m$ of the Block $\mathrm{B}$, is only considered while disregarding $C$, considering that as a disturbance.

\section{4. $P C C V_{P}$ controller with a proportional gain and an integrator as the compen- sator}

A suitable compensator for the PCC voltage controller should be chosen. The PCC $V_{P}$ controller shown in Fig. 4 and the PCC $V_{Q}$ controller shown in Fig. 3(a) demonstrate similar dynamic characteristics. The only difference between the two control loops is the static gains. Therefore, an integrator with a gain identified as a suitable compensator for the PCC $V_{Q}$ controller can be applied in the PCC $V_{P}$ controller as well.

The closed-loop transfer function of the PCC $V_{P}$ controller with the chosen compensator can be derived as,

$$
G_{V_{\mathrm{gPCli}}}(s)=\frac{\frac{2 m k_{\mathrm{pp}}}{V_{\mathrm{gm}} s} G_{V_{\mathrm{gP}}}(s)}{1+\frac{2 m k_{\mathrm{pp}}}{V_{\mathrm{gm}} s} G_{V_{\mathrm{gP}}}(s)},
$$

where $k_{\mathrm{pp}}$ is the gain of the integrator. Equation (7) can be simplified as,

$$
G_{V_{\mathrm{gPcli}}}(s)=\frac{1}{\left(\frac{1}{K_{\mathrm{pp}} R_{\mathrm{g}}}\right) s+1},
$$

where

$$
K_{\mathrm{pp}}=\frac{2 m k_{\mathrm{pp}}}{V_{\mathrm{gm}}} .
$$

As per (8), the dynamic performance that determines the time constant of the PCC $V_{P}$ controller, depends on the gain of the controller as well as the grid impedance that is seen by the PV system at the PCC. The time constant of $G_{V_{\mathrm{gPcli}}}(s)$ is set to $0.2 \mathrm{~s}$ when the grid impedance is $(0.4+\mathrm{j} 0.25) \Omega$. The chosen time constant for the closed-loop PCC $V_{P}$ controller is high enough to decouple this controller from 
the rest of the control system that is associated with the active power transfer of the PV system. With the chosen time constant for the PCC voltage controller,

$$
\left(\frac{1}{K_{\mathrm{pp}} R_{\mathrm{g}}}\right)=0.2
$$

and if the reference grid impedance is applied,

$$
K_{\mathrm{pp}}=12.5 \text {. }
$$

For the given operating conditions of the PV array illustrated by Fig. $5, m=$ $-70 \mathrm{~W} / \mathrm{V}$. Further $V_{\mathrm{gm}}=\sqrt{2} U_{\max } \mathrm{V}$. Hence,

$$
k_{\mathrm{pp}}=32 .
$$

The performance of the PCC $V_{P}$ controller is evaluated by integrating the PCC $V_{Q}$ controller with the simulation model of the PV system in [19]. In this simulation study, the PCC voltage of the PV system was controlled only by the PCC $V_{P}$ controller. Hence reactive power controllers were disabled. The PCC voltage reference, $V_{\mathrm{gm} 0}$ was set to $\sqrt{2} U_{\max }$. Simulation results of the PCC voltage controller are shown in Fig. 7. Initially the PCC $V_{P}$ controller was deactivated and at $t=1 \mathrm{~s}$, the controller was activated. As shown in Fig. 7(a), the controller was able to regulate the PCC voltage at the reference voltage by curtailing approximately $1.8 \mathrm{~kW}$. The steady-state has been reached within $1 \mathrm{~s}$.

Since the time constant of the PCC voltage controller is chosen as $0.2 \mathrm{~s}$, the steady state should be attained within $1 \mathrm{~s}$ after a disturbance. The simulation results verify this performance criteria and hence the design criteria of the PCC $V_{P}$ controller. Further, the dynamic response of the PCC $V_{P}$ controller is slightly different to the dynamic response of a first order system. This deviation of the response is due to the linear approximation of the characteristic curve of the PV array that was made in deriving the expression for Block B in Fig. 4.

\section{Advanced PCC voltage control strategies for PV systems}

The PCC voltage of a grid-connected PV system can be regulated at a set reference voltage with dynamic active and reactive power controllers, namely the PCC $V_{Q}$ controller and the PCC $V_{P}$ controller discussed in previous sections. If real power is curtailed to regulate the PCC voltage of the PV system as is achieved using the PCC $V_{P}$ controller, two different operating strategies that combine the operation of the PCC $V_{Q}$ controller and the PCC $V_{P}$ controller can be proposed.

\subsection{Fixed minimum lagging power factor operation}

The minimum lagging power factor at which a grid-connected energy system via an inverter should be operated is specified as 0.95 in AS4777.2 [4]. Even if the active power is curtailed to regulate the PCC voltage of the PV system, by adhering to the standard, the minimum lagging power factor of the PV system can be maintained at 0.95 and such an operation of the PV system is described in this section. 


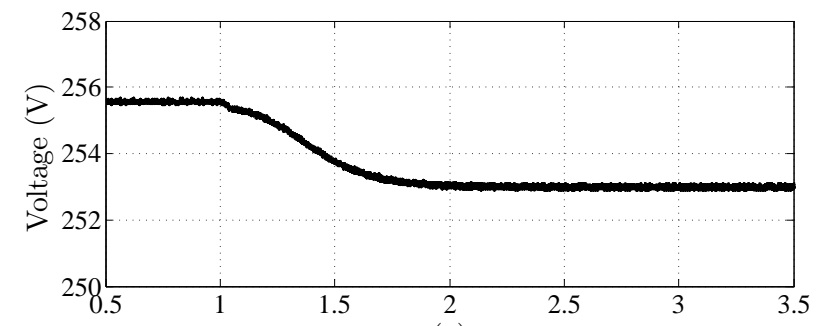

(a)

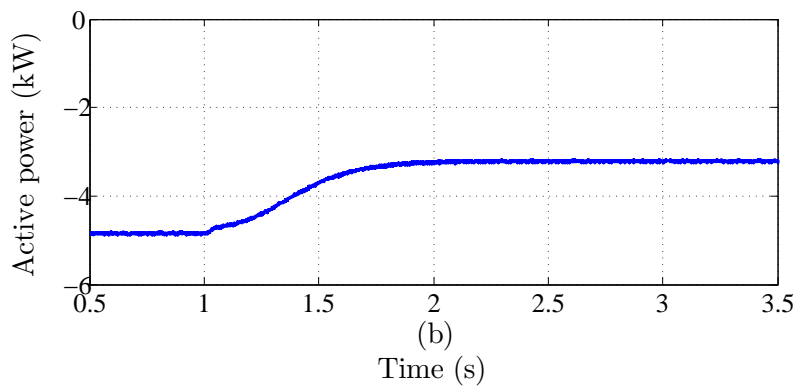

Figure 7: Dynamic performance of the PCC $V_{P}$ controller; (a) rms voltage of the PCC $\left(V_{\mathrm{g}}\right)$ and (b) active power injected to the grid $\left(P_{\mathrm{g}}\right)$ by the PV system.

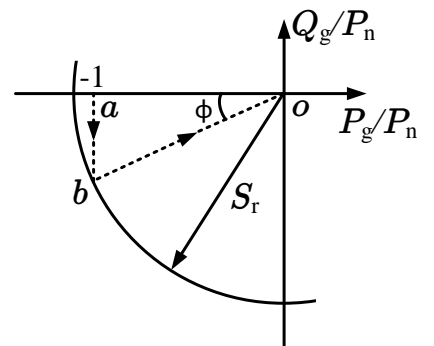

(a)

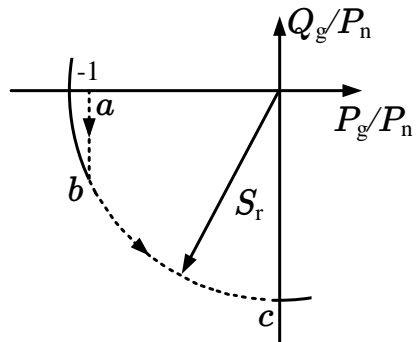

(b)

Figure 8: Novel operating strategies for a PV system; (a) fixed minimum power factor operation and (b) fixed maximum apparent power operation.

The fixed minimum power factor operation of a PV system is illustrated in Fig. 8(a). Here, $S_{\mathrm{r}}$ is the rated apparent power capacity of the VSC that interfaces the PV system to the power distribution grid and the lagging power factor, $\cos (\phi)=0.95$. At a given time, the active power injected to the grid by the PV system is $P_{\mathrm{g}}$. At the operating point $a$, the $\mathrm{PV}$ system is injecting the nominal power, $P_{\mathrm{n}}$, of the PV system to the grid and hence $P_{\mathrm{g}} / P_{\mathrm{n}}=-1$. The PCC $V_{Q}$ controller is active from $a$ to $b$, and the controller saturates at $b$ as a result of the PV system reaching the minimum lagging power factor limit. After $b$ the MPPT of the $\mathrm{PV}$ system is disabled and the PCC $V_{P}$ controller is activated. The operating point of the PV system moves towards $o$ from $b$ until the PCC voltage is regulated at the reference voltage. Though the PCC $V_{Q}$ controller is active from $b$ to $o$, it does not contribute to dynamic PCC voltage regulation actively since the controller is saturated.

The operation of the PV system at a minimum lagging power factor is ensured by the fixed minimum power factor operation of the PV system. In this operation mode, the full capacity of the PV system may not be used most of the time. Further, 
when the PCC $V_{P}$ controller is enabled, in order to maintain the minimum lagging power factor, the reactive power absorbed from the grid is reduced in proportion to the amount of active power curtailed. The reduction in the amount of reactive power absorbed by the PV system causes further active power curtailment in order to regulate the $\mathrm{PCC}$ voltage at the reference level.

\subsection{Fixed maximum apparent power operation}

The purpose of defining a minimum power factor in the Australian Standard AS4777.2 [4] is to minimise the reactive power flow in the grid in order to reduce the network losses and to avoid overloading the distribution transformer in a large installation of grid-connected energy systems via inverters. The weakest nodes in the power distribution grid are the most sensitive nodes to voltage variations and are the most effective nodes to regulate the voltage. If the reference voltages of dynamic active and reactive power controllers are properly selected, the PV systems at weak nodes may start regulating the respective PCC voltage before other PV systems in the grid. If the PCC voltage regulation is enabled without limiting the reactive power absorption to a minimum lagging power factor operation but limiting to the available capacity of the VSC, the PV systems at the strong nodes of the grid may not need to contribute to the voltage regulation of the network. But the PV systems at weak nodes may contribute to voltage regulation of the grid utilising the rated capacity of such systems. Hence reactive power flow in the network may be minimised.

The fixed maximum apparent power operation of a PV system is illustrated in Fig. 8(b). The operation of the PV system from $a$ to $b$ is same as that is described in Section 5.1. At $b$, the PCC $V_{Q}$ controller is saturated and the PCC $V_{P}$ controller is activated. Unlike fixed minimum power factor operation, when power is curtailed to regulate the PCC voltage of the PV system, a proportional amount of reactive power is absorbed by the PV system. Hence, after $b$, active power curtailment as well as the additional reactive power absorbed by the PV system contribute to PCC voltage regulation. Though the PCC $V_{Q}$ controller is enabled after $b$, that controller does not contribute to voltage regulation actively since the controller is saturated.

Since the full power capacity of the VSC that interfaces the PV system to the grid is used, the active power curtailment to regulate the PCC voltage in the fixed maximum apparent power operation is less compared to the fixed minimum power factor operation of the PV system. In fixed minimum power factor operation, the PV system may disconnect from the grid after curtailing active power up to a certain level. However, in fixed maximum apparent power operation the PV system stays connected to the grid even though injecting no active power while absorbing reactive power to regulate the $\mathrm{PCC}$ voltage.

\section{Experimental results}

The dynamic performance of the PCC $V_{Q}$ controller and the PCC $V_{P}$ controller, and also the feasibility of implementing the novel operating strategies proposed for a PV system in this paper, were experimentally verified and the results are presented and discussed in this Section. 


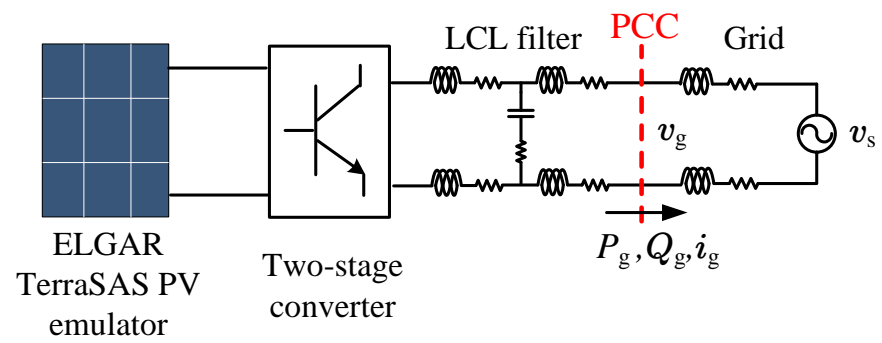

Figure 9: Simplified block diagram of the experimental setup.

An experimental setup of a single-phase, grid-connected, two-stage PV system established in the laboratory was used to obtain test results. A schematic diagram of the experimental setup is shown in Fig. 9. A detailed simulation model of the implemented experimental setup can be found in [19].

An ELGAR Terra SAS PV array emulator was used for the PV system. A California Instruments MX30 AC and DC power source in combination with OMNI $3-75$ impedance bank was used to simulate the power distribution grid. The impedance of the simulated grid was $(0.25+j 0.25) \Omega$. Each practical result obtained using an oscilloscope and presented in this section consists of 4 waveforms; (1) the rms voltage at the PCC of the PV system, $V_{\mathrm{g}}(2)$ reactive power injected to the grid, $Q_{\mathrm{g}}(3)$ active power injected to the grid, $P_{\mathrm{g}}$ and $(4)$ the current injected to the grid by the PV system, $i_{\mathrm{g}}$. The first three quantities, $V_{\mathrm{g}}, Q_{\mathrm{g}}$ and $P_{\mathrm{g}}$ were obtained through the controller and displayed on the oscilloscope via $\mathrm{D} / \mathrm{A}$ conversion channels. The ripple on the waveform of $V_{\mathrm{g}}$ is an artifact of the A/D conversion of the measured PCC voltage.

In the controller of the experimental PV system, the voltage reference of the PCC $V_{Q}$ controller and the PCC $V_{P}$ controller were set as $\sqrt{2} U_{\max }$. The practical results showing the dynamic performance of the PCC $V_{Q}$ controller and PCC $V_{P}$ controller and also the proposed novel operating strategies were obtained by introducing a step voltage change in the grid that causes the PCC voltage of the PV system to rise above $\sqrt{2} U_{\max }$.

As per (1), the dynamic performance of the PCC $V_{Q}$ controller depends on the reactance, $X_{\mathrm{g}}$, but not on the resistance, $R_{\mathrm{g}}$, of the grid impedance seen by the PV system at the PCC. The reactance of the grid impedance as used in the simulation and experimental setup is similar. Hence, the gain of the integrator as used in the simulation work presented in Section 3.2 was used in the controller of the experimental PV system so that the steady-state of the PCC $V_{Q}$ controller is reached within $1 \mathrm{~s}$.

The dynamic performance of the PCC $V_{Q}$ controller is shown in Fig. 10(a). In this experiment the PCC $V_{P}$ controller was disabled. According to Fig. 10(a), the PCC $V_{Q}$ controller has been activated once $V_{\mathrm{g}}$ rises above $U_{\max }$, i.e. $253 \mathrm{~V}$. After the step voltage change in the grid, the PCC voltage is seen to be regulated at the reference level by absorbing approximately $1.75 \mathrm{kVAr}$ of reactive power and the steady-state has been reached within approximately $1 \mathrm{~s}$.

Unlike in the case of the PCC $V_{Q}$ controller, the dynamic performance of the PCC $V_{P}$ controller depends primarily on the resistance of the grid impedance, $R_{\mathrm{g}}$, 


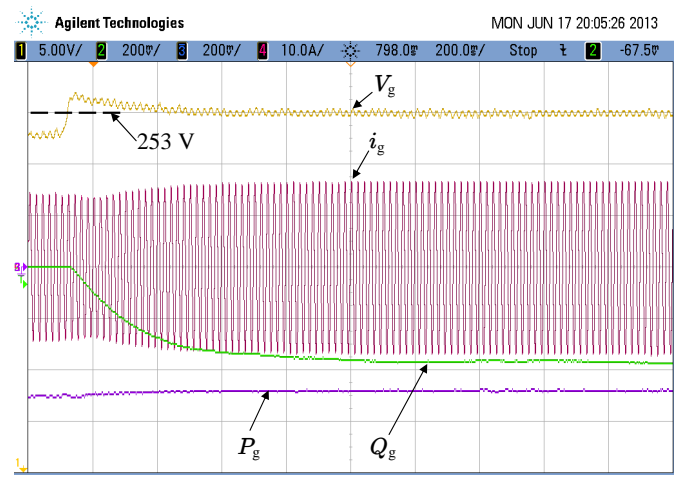

(a) Performance of the PCC $V_{Q}$ controller

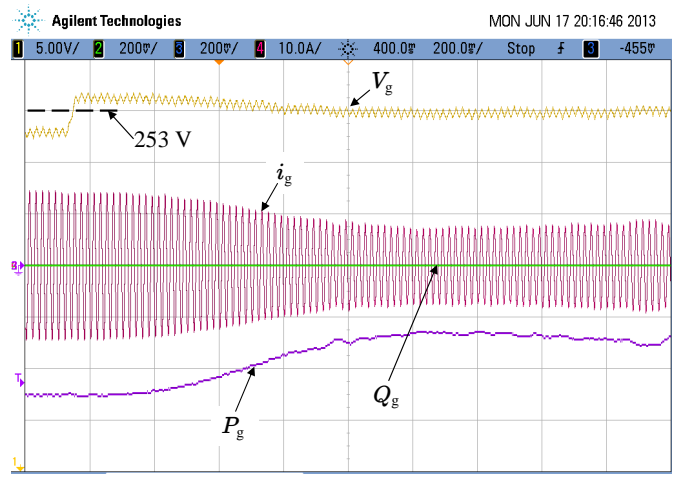

(b) Performance of the PCC $V_{P}$ controller

Figure 10: Performance of the PCC $V_{Q}$ and $V_{P}$ Controllers: Ch1: rms voltage of the PCC $\left(V_{\mathrm{g}}\right)$ [5 V/div] Ch2: reactive power injected to the grid $\left(Q_{\mathrm{g}}\right)[1 \mathrm{kVAr} / \mathrm{div}]$, Ch3: active power injected to the grid $\left(P_{\mathrm{g}}\right)[1 \mathrm{~kW} / \mathrm{div}]$, Ch4: current injected to the grid $\left(i_{\mathrm{g}}\right)[10 \mathrm{~A} / \mathrm{div}]$.

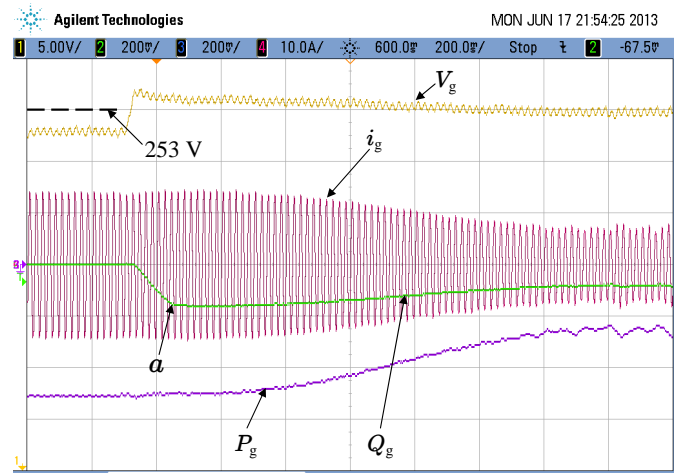

(a) Fixed minimum lagging power factor operation of the PV system

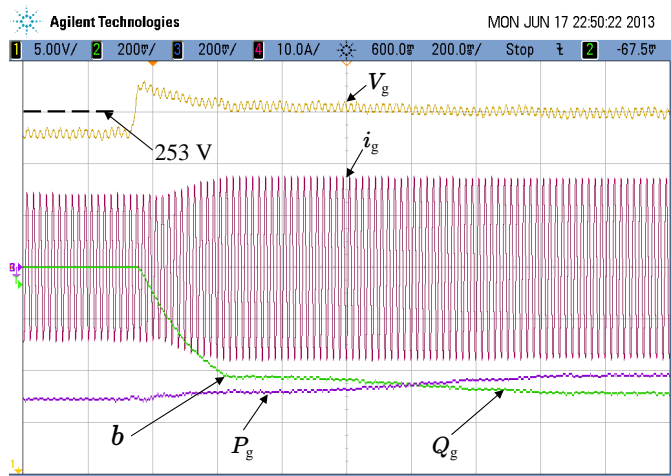

(b) Fixed maximum apparent power operation of the PV system

Figure 11: Performance of the PV system under different operating modes: Ch1: rms voltage of the PCC $\left(V_{\mathrm{g}}\right)[5 \mathrm{~V} / \mathrm{div}] \mathrm{Ch} 2$ : reactive power injected to the grid $\left(Q_{\mathrm{g}}\right)[1 \mathrm{kVAr} / \mathrm{div}]$, Ch3: active power injected to the grid $\left(P_{\mathrm{g}}\right)[1 \mathrm{~kW} / \mathrm{div}]$, Ch4: current injected to the grid $\left(i_{\mathrm{g}}\right)[10 \mathrm{~A} / \mathrm{div}]$.

as per (4). The resistance of the grid impedance in the simulation work presented in Section 4.4 and the experimental PV system are different in value. Therefore the gain of the integrator of the PCC $V_{P}$ controller, $k_{\mathrm{pp}}$ is calculated as 51 to obtain the steady-state response of the PCC $V_{P}$ controller of the experimental PV system within $1 \mathrm{~s}$, assuming the characteristics of the PV array are as shown in Fig. 5.

Fig. 10(b) illustrates the dynamic performance of the PCC $V_{P}$ controller. In this experiment the PCC $V_{Q}$ controller was disabled. According to Fig. 10(b), after the step change in voltage of the grid that causes the PCC voltage to rise above $U_{\max }$, the PCC $V_{P}$ controller has been able to regulate the PCC voltage at $U_{\max }$ by curtailing approximately $1 \mathrm{~kW}$ of active power. The steady-state has been reached within approximately $1 \mathrm{~s}$. As shown, the smoothness of the $P_{\mathrm{g}}$ variation has been degraded when $P_{\mathrm{g}}$ is curtailed to regulate the PCC voltage. This behaviour is introduced by the PV emulator in combination with the power electronic converter used in the experimental PV system since the emulator is unable to maintain a steady operating 
point at lower power levels of the characteristic curve. The variation of $P_{\mathrm{g}}$ as shown in Fig. 10(b) is comparable with that of Fig. 7(b).

The fixed minimum power factor operation and the fixed maximum apparent power operation of the experimental PV system are illustrated in Fig. 11(a) and Fig. 11(b) respectively. In the respective operating mode, the minimum power factor was set as 0.95 lagging and the maximum apparent power of the VSC was limited to $3.2 \mathrm{kVA}$. In both operating modes, initially the PCC $V_{Q}$ controller was triggered upon the PCC voltage rising above $U_{\max }$. Then that controller saturated upon reaching the reactive power limit specified by the operating mode at $a$ and $b$ in Fig. 11(a) and Fig. 11(b) respectively. In both operating modes, the PCC $V_{P}$ controller was triggered when the PCC $V_{Q}$ controller was saturated and active power curtailment commenced in order to regulate the PCC voltage at $U_{\max }$. As illustrated in Fig. 11(b) in the fixed maximum apparent power operation of the PV system, the PCC voltage can be regulated by curtailing a lower amount of active power and obviously absorbing a larger amount of reactive power from the grid, unlike in the fixed minimum power factor operation of the PV system as illustrated in Fig. 11(a). In steady-state, the PV systems was injecting about $1.2 \mathrm{~kW}$ to the grid while absorbing only about $0.4 \mathrm{kVAr}$ from the grid in the fixed minimum lagging power factor operation and injecting about $2.1 \mathrm{~kW}$ to the grid and absorbing about $2.5 \mathrm{kVAr}$ from the grid.

\section{Conclusions}

The proposed closed-loop PCC voltage controllers in this paper are able to regulate the PCC voltage of a PV system at a reference level by dynamically controlling the active or reactive power response of the PV system. Simulation results and experimental validations presented in this paper prove that the controller design procedures illustrated in this paper are accurate and the response of the controllers is predictable. The fixed minimum power factor operation that is proposed by combining closed-loop PCC voltage controllers minimises the reactive power consumption while staying connected to the grid until the power is curtailed to a specified level. Unlike in the fixed minimum power factor operation, the maximum apparent power operation proposed in the paper may utilise the full capacity of the converter that interfaces the PV system to the grid. Therefore, with the maximum apparent power operation of a PV system, PCC voltage regulation may be achieved with minimum active power curtailment. Further, the maximum apparent power operation the PV system may provide voltage support with reactive power even without injecting any active power to the grid.

The closed-loop PCC voltage controllers discussed in this paper will lead to effective and robust PCC voltage regulation of a PV system with both active and reactive power. Further, the novel operation strategies proposed will enable effective utilisation of PV systems in order to contribute to grid voltage control.

\section{Acknowledgement}

The authors greatly acknowledge the invaluable discussions had with Douglas Carter (DenKinetic Pty Ltd, Australia) and Sridhar R. Pulikanti (ABB Limited, 
New Zealand) and also the support received from them while implementing the experimental setup of the single-phase grid-connected, two-stage solar photovoltaic (PV) system.

[1] T. Stetz, W. Yan, M. Braun, Voltage control in distribution systems with high level PV-penetration -improving absorption capacity for PV systems by reactive power supply, in: Proc. 25th European Photovoltaic Solar Energy Conf. and Exhibition, 2010, pp. 5000-5006.

[2] E. Demirok, et al., Local reactive power control methods for overvoltage prevention of distributed solar inverters in low-voltage grids, IEEE Trans. Photovoltaics 1 (2) (2011) 174-182.

[3] T. Stetz, F. Marten, M. Braun, Improved low voltage grid-integration of photovoltaic systems in Germany, IEEE Trans. Sustain. Energy 4 (2) (2013) 534-542.

[4] Grid connection of energy systems via inverters - part 2: inverter requirements, Tech. Rep. AS4777.2, Australian Standards (2005).

[5] S. B. Kjaer, J. K. Pedersen, F. Blaabjerg, A review of single-phase gridconnected inverters for photovoltaic modules, IEEE Trans. Ind. Appl. 41 (5) (2005) 1292-1306.

[6] T. Kerekes, M. Liserre, R. Teodorescu, C. Klumpner, M. Sumner, Evaluation of three-phase transformerless photovoltaic inverter topologies, IEEE Trans. Power Electron. 24 (9) (2009) 2202-2211.

[7] G. Kerber, R. Witzmann, H. Sappl, Voltage limitation by autonomous reactive power control of grid connected photovoltaic inverters, in: Proc. Compatibility and Power Electronics (CPE '09), 2009, pp. 129-133. doi:10.1109/CPE.2009.5156024.

[8] S. Abbott, B. Fox, D. Morrow, Distribution network voltage support using sensitivity-based dispatch of distributed generation, in: Power and Energy Society General Meeting (PES), 2013 IEEE, 2013, pp. 1-5. doi:10.1109/PESMG.2013.6672148.

[9] X. Su, M. A. S. Masoum, P. J. Wolfs, optimal pv inverter reactive power control and real power curtailment to improve performance of unbalanced four-wire lv distribution netowrks, IEEE Trans. Sustain. Energy 5 (3) (2014) 967-977.

[10] J. Jung, A. Onen, R. Arghandeh, R. P. Broadwater, Coordinated control of automated devices and photovoltaic generators for voltage rise mitigation in power distribution circuits, Renewable Energy 66 (0) (2014) 532 - 540. doi:http://dx.doi.org/10.1016/j.renene.2013.12.039.

URL http://www.sciencedirect.com/science/article/pii/ S096014811400010X 
[11] E. Demirok, D. Sera, P. Rodriguez, R. Teodorescu, Enhanced local grid voltage support method for high penetration of distributed generators, in: Proc. 37th Annual Conf. of the IEEE Industrial Electronics Society (IECON'11), 2011, pp. 2481-2485. doi:10.1109/IECON.2011.6119699.

[12] T. Tran-Quoc, T. Le, C. Kieny, N. Hadjsaid, S. Bacha, C. Duvauchelle, A. Almeida, Local Voltage Control of PVs in Distribution Networks, in: Electricity Distribution - Part 1, 2009. CIRED 2009. 20th International Conference and Exhibition on, 2009, pp. 1-4.

[13] E. Demirok, Others, Evaluation of the voltage support strategies for the low voltage grid connected PV generators, in: Proc. IEEE Energy Conversion Congress and Exposition (ECCE), 2010, pp. 710-717.

[14] R. Tonkoski, L. A. C. Lopes, T. H. M. El-Fouly, Coordinated active power curtailment of grid connected PV inverters for overvoltage prevention, IEEE Trans. Sustain. Energy 2 (2) (2011) 139-147.

[15] R. Tonkoski, L. A. Lopes, Impact of Active Power Curtailment on Overvoltage Prevention and Energy Production of PV Inverters Connected to Low Voltage Residential Feeders, Renewable Energy 36 (12) (2011) 3566 - 3574. doi:http://dx.doi.org/10.1016/j.renene.2011.05.031.

URL http://www.sciencedirect.com/science/article/pii/ S096014811100259X

[16] Electromagnetic Compatibility (EMC) - Consideration of reference impedance and public supply network impedances for use in determining disturbances characteristics of electrical equipment having a rated current $\leq 75$ A per phase, Tech. Rep. TR 60725, IEC (Jun. 2012).

[17] B. K. Perera, P. Ciufo, S. Perera, Point of common coupling (PCC) voltage control of a grid-connected solar photovoltaic system, in: Proc. 39th Annual Conf. of the IEEE Industrial Electronics Society (IECON'13), 2013, pp. 1-6.

[18] A. Yazdani, R. Iravani, Voltage-Sourced Converters in Power Systems, Wiley/IEEE, 2010.

[19] B. K. Perera, S. R. Pulikanti, P. Ciufo, S. Perera, Simulation model of a grid-connected single-phase photovoltaic system in PSCAD/EMTDC, in: Proc. IEEE Int. Conf. on Power System Technology (POWERCON), 2012, pp. 1-6. 